\title{
Synthesis and antimicrobial activity of novel oxime derivatives of phenothiazine
}

\author{
Ashutosh Barvea, *, Malleshappa Noolvi ${ }^{b}$, Niharika Subhedara, Vishnu Dev Guptaa and Gaurav Bhatiac \\ a Department of Pharmaceutical Chemistry, Bhanwar Nahata College of Pharmacy, Mandsaur, 458001, India \\ b Department of Pharmaceutical Chemistry, Amar Shaheed Baba Ajit Singh Jujhar Singh Memorial College of Pharmacy, Bela, 140111, India \\ c Department of Pharmacognosy, Smriti College of Pharmaceutical Education, Indore, 452001, India \\ ${ }^{*}$ Corresponding author at: Department of Pharmaceutical Chemistry, Bhanwar Nahata College of Pharmacy, Mandsaur, 458001, India. Tel.: +91.9893107417; \\ fax: +91.1881263655. E-mail address: ashutoshbarve@ymail.com (A. Barve).
}

\section{ARTICLE INFORMATION}

Received: XX November 2010

Received in revised form: XX January 2011

Accepted: XX January 2011

Online: 30 September 2011

\section{KEYWORDS}

\section{Coumarin}

Oxime

Phenothiazine

Antimicrobial activity

Anti-bacterial activity

Anti-fungal activity

\section{Introduction}

In recent times microbial resistance against antimicrobial agents has increased remarkably. New prototype compounds are required to deal with this problem, so discovery of novel synthetic and semi-synthetic product as antimicrobials is the prerequisite of present health scenario, and continuous effort in development of same is very much required. Pharmacological properties of coumarin aroused our interest to explore new analogs of coumarin for antimicrobial activity. Coumarin and its analogues are widely distributed in plants and they are responsible for wide variety of the activities especially antibacterial and antifungal [1-10]. The in vitro antimicrobial activity of phenothiazine was first described by Paul Ehlich early in the twentieth century [11]. Various studies show and support those phenothiazines may be used for the management of bacterial or fungal infections [12-21]. The study also shows that phenothiazine not only itself having antimicrobial effect but also it show synergistic interaction with other antimicrobial [22]. Moreover various heterocyclic compounds containing oxime, and the complexes of oximes with different transition metals are reported in the literature and. Found: to be active as antibacterial, antitubercular, antilepral, antiviral and antimalarial [23-34] so we consider these reports and incorporate this functional moiety into our scheme. Therefore the development of facile synthetic routes to achieve access to these molecules is of prime interest. In view of the above mentioned pharmacological applications of phenothiazine coumarin and oxime and in continuation of our research on the synthesis of biologically active molecules, we considered undertaking the design and synthesis of hitherto unknown phenothiazine derivatives. Further, the increasing number of multidrug resistant pathogens has led us to screen the newly synthesized derivatives against the representative panel of Gram-positive [Gr (+)] and Gram-negative [Gr (-)] bacteria and fungi.

\section{Experimental}

\subsection{Instrumentation}

Melting points were determined in Thermonik melting point apparatus and are uncorrected. IR spectrum was recorded on Thermonicolet FT-IR 200 spectrophotometer by using $\mathrm{KBr}$ pellet values are expressed in $\mathrm{cm}^{-1}$. NMR spectra were recorded in DMSO- $d_{6}$ using varian $400 \mathrm{MHz}$ mercury plus and chemical shift are reported in $\delta$ (ppm). Mass spectra were recorded on GCMS-QP 2010 Shimaduzu and mass values are reported in $\mathrm{m} / \mathrm{z}$. All the chemicals used are of reagent grade and substituted aromatic amines and other chemicals used during synthesis are of synthesis fine grade.

\subsection{Synthesis}

The synthesis of 7-hydroxy-4-methylcoumarin (I) was carried out by the reaction of resorcinol and ethylacetoacetate; it is well established [35]. The various substituted 7-arylamino4-methyl-coumarin (IIa-j) were prepared by reacting with substituted aromatic amines (a-j). The 7-arylamino-4-methylcoumarins (IIa-j) were further converted into 4-methyl-2-oxopyranyl-phenothiazines (IIIa-j) by the action of sulphur in presence of iodine. Further these novel 4-methyl-2-oxopyranyl-phenothiazines (IIIa-j) derivatives, on treatment with hydroxylamine hydrochloride were converted to 4- 
methylpyrano-(2,3- $\beta$ )-phenothiazine-2(11h)-one oxime (IVa-j) derivatives as shown in Scheme 1. The reaction and purity of compounds were monitored by TLC using precoated silica gel. structures of the compounds were confirmed by spectral studies.

\subsubsection{Preparation of compounds (IIIa-j) [35,36]}

In $50 \mathrm{~mL}$ beaker, mixture of resorcinol (3.7 g) and ethylacetoacetate $(4.4 \mathrm{~mL})$ was added to concentrated sulphuric acid $(15 \mathrm{~mL})$ at $5{ }^{\circ} \mathrm{C}$ with constant stirring for 30 minutes. Mixture was poured on to the crushed ice (about 100 g), with vigorous stirring, 7-hydroxy-4-methyl coumarin (I) was precipitated. Suspension was filtered; crude was dissolved in cold aq. sodium hydroxide $(10 \%)$ solution and reprecipitated it by addition of dilute hydrochloric acid. Crude was decolorized and re-crystallized from charcoal and ethanol respectively [35]. A mixture of 7-hydroxy-4-methyl coumarin (I) $(1 \mathrm{~mol})$ and primary aromatic amine $(1 \mathrm{~mol})$ in absolute ethanol $(20 \mathrm{~mL})$ was heated under reflux in presence of anhydrous $\mathrm{ZnCl}_{2}(0.2 \mathrm{~g})$ for $5 \mathrm{~h}$. It was cooled and the separated crude mass was filtered, washed repeatedly with cold water, dried and recrystallized from methanol, the synthesized 7arylamino-4-methyl-coumarin (IIa-j) and sulphur powder (1.0 g) were heated together at $150-160^{\circ} \mathrm{C}$ for $3 \mathrm{~h}$ in the presence of iodine $(150 \mathrm{mg})$. The mixture was cooled to room temperature and treated with dilute hydrochloric acid $(100 \mathrm{~mL})$ to remove unreacted amine and washed repeatedly with warm water. Residue was dried in vacuum to yield phenothiazine analogs (IIIa-j) [36].

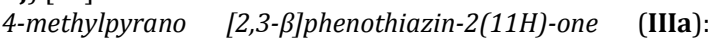
Yield: 54.5\%. M.p.: 180-182 ${ }^{\circ} \mathrm{C}$. FT-IR $\left(\mathrm{KBr}, \mathrm{cm}^{-1}\right): 3446 v(\mathrm{~N}-\mathrm{H})$, $3101 v(\mathrm{C}-\mathrm{H}), 2950 v(\mathrm{C}-\mathrm{H}), 1738 v(\mathrm{C}=0)$ (pyraneone), $1571 v(\mathrm{C}-$ N) (hetero), $1450 v(\mathrm{C}=\mathrm{C}), 665 v(\mathrm{C}-\mathrm{S})$ (hetero). ${ }^{1} \mathrm{H}$ NMR $(400$ MHz, DMSO- $\left.d_{6}, \delta, \mathrm{ppm}\right): 6.92(\mathrm{~s}, 1 \mathrm{H}, \mathrm{Ar}-H), 6.30-6.88(\mathrm{~m}, 4 \mathrm{H}$, Ar- $H$ ), 6.26 (s, 1H, Ar- $H$ ), 5.67 (s, 1H, CO-CH), 4.17 (s, 1H, C-NHC), $1.70\left(\mathrm{~s}, 3 \mathrm{H}, \mathrm{C}-\mathrm{CH}_{3}\right) \cdot{ }^{13} \mathrm{C}$ NMR $\left(400 \mathrm{MHz}, \mathrm{CDCl}_{3}, \delta, \mathrm{ppm}\right): 160.9$ $(1 \mathrm{C},>\mathrm{C}=0), 152.8-110.1(14 \mathrm{C}, \mathrm{Ar}-\mathrm{C}), 21.3$ (1C, C-CH $). \mathrm{MS}$ (DIPMS, m/z): 280.05 and HMS calculated for $\mathrm{C}_{16} \mathrm{H}_{11} \mathrm{NO}_{2} \mathrm{~S}$ : 281.051. Found: 281.0519.

10-hydroxy-4-methylpyrano [2,3- $\beta]$ phenothiazin-2(11H)one (IIIb): Yield: 59\%. M.p.: 182-184 ${ }^{\circ} \mathrm{C}$. FT-IR $\left(\mathrm{KBr}, \mathrm{cm}^{-1}\right): 3453$ $v(\mathrm{~N}-\mathrm{H})$ (hetero), $3350 v(\mathrm{O}-\mathrm{H})$ (phenol), $3037 v(\mathrm{C}-\mathrm{H}), 2877 v(\mathrm{C}-$ $\mathrm{H})$ (aliphatic), $1741 v(\mathrm{C}=0)$ (pyraneone), $1525 \mathrm{v}(\mathrm{C}-\mathrm{N})$ (hetero), $1456 v(\mathrm{C}=\mathrm{C})$ (aromatic), $1263 v(\mathrm{C}-0)$ (aromatic), $702 v(\mathrm{C}-\mathrm{S})$ (hetero). ${ }^{1} \mathrm{H}$ NMR $\left(400 \mathrm{MHz}, \mathrm{DMSO}-d_{6}, \delta, \mathrm{ppm}\right): 6.30-7.02(\mathrm{~m}$, $5 \mathrm{H}, \mathrm{Ar}-\mathrm{H}), 5.73$ (s, 1H, CO-CH), 5.00 (s, 1H, Ar- $\mathrm{OH}), 4.13$ (s, 1H, $\mathrm{C}-\mathrm{NH}-\mathrm{C}), 1.78\left(\mathrm{~s}, 3 \mathrm{H}, \mathrm{C}-\mathrm{CH}_{3}\right) .{ }^{13} \mathrm{C}$ NMR ( $\left.400 \mathrm{MHz}, \mathrm{CDCl}_{3}, \delta, \mathrm{ppm}\right)$ : $161.1(1 \mathrm{C},>C=0), 152.5-109.2(14 \mathrm{C}, \mathrm{Ar}-\mathrm{C}), 21.2\left(1 \mathrm{C}, \mathrm{C}-\mathrm{CH}_{3}\right)$. MS (DIPMS, m/z): $296.04\left(\mathrm{M}^{+}\right)$and HMS calculated for $\mathrm{C}_{16} \mathrm{H}_{11} \mathrm{NO}_{3} \mathrm{~S}$ : 297.046. Found: 297.0456.

9-hydroxy-4-methylpyrano [2,3- $\beta]$ phenothiazin-2(11H)-one (IIIc): Yield: $45.5 \%$. M.p.: $178-180^{\circ} \mathrm{C}$. FT-IR $\left(\mathrm{KBr} \mathrm{cm}^{-1}\right): 3108$ $v(\mathrm{C}-\mathrm{H})$ (aromatic), $2866 \mathrm{v}(\mathrm{C}-\mathrm{H})$ (aliphatic), $1446 v(\mathrm{C}=\mathrm{C})$ (aromatic), $1483 v(\mathrm{C}-\mathrm{N})$ (hetero), $3300 \mathrm{v}(\mathrm{N}-\mathrm{H})$ (hetero), 1716 $v(\mathrm{C}=\mathrm{O})$ (pyraneone), $1242 v(\mathrm{C}-\mathrm{O})$ (aromatic), $3218 v(\mathrm{O}-\mathrm{H})$ (phenol), $669 v(\mathrm{C}-\mathrm{S})$ (hetero). ${ }^{1} \mathrm{H}$ NMR $\left(400 \mathrm{MHz}, \mathrm{DMSO}-d_{6}, \delta\right.$, ppm): 6.35-7.13 (m, 5H, Ar- $H$ ), $5.87(\mathrm{~s}, 1 \mathrm{H}, \mathrm{CO}-\mathrm{CH}), 5.06(\mathrm{~s}, 1 \mathrm{H}$, $\mathrm{Ar}-\mathrm{OH}), 4.17$ (s, $1 \mathrm{H}, \mathrm{C}-\mathrm{N} H-\mathrm{C}), 1.07\left(\mathrm{~s}, 3 \mathrm{H}, \mathrm{C}-\mathrm{CH}_{3}\right) .{ }^{13} \mathrm{C}$ NMR $(400$ $\left.\mathrm{MHz}, \mathrm{CDCl}_{3}, \delta, \mathrm{ppm}\right): 160.4(1 \mathrm{C},>C=0), 151.3-111.5$ (14C, Ar-C), $21.6\left(1 \mathrm{C},-\mathrm{CH}_{3}\right)$. MS (DIPMS, m/z): $296.04\left(\mathrm{M}^{+}\right)$and HMS calculated for $\mathrm{C}_{16} \mathrm{H}_{11} \mathrm{NO}_{3} \mathrm{~S}: 297.046$. Found: 297.0456.

8-hydroxy-4-methylpyrano[2,3-ß]phenothiazin-2(11H)-one (IIId): Yield: 50\%. M.p.: 176-179 ${ }^{\circ} \mathrm{C}$. FT-IR $\left(\mathrm{KBr}, \mathrm{cm}^{-1}\right): 3477$ $v(\mathrm{O}-\mathrm{H}) \quad$ (phenol), $3356 \quad v(\mathrm{~N}-\mathrm{H})$ (hetero), $3049 \quad v(\mathrm{C}-\mathrm{H})$ (aromatic), $2867 v(\mathrm{C}-\mathrm{H})$ (aliphatic), $1720 v(\mathrm{C}=0)$ (pyraneone), $1510 v(\mathrm{C}-\mathrm{N})$ (hetero), $1460 v(\mathrm{C}=\mathrm{C})$ (aromatic), $1230 v(\mathrm{C}-\mathrm{O})$ (aromatic), $675 v(\mathrm{C}-\mathrm{S})$ (hetero). ${ }^{1} \mathrm{H}$ NMR $\left(400 \mathrm{MHz}\right.$, DMSO- $d_{6}, \delta$, ppm): 6.31-7.01 (m, 5H, Ar- $H$ ), $5.64(\mathrm{~s}, 1 \mathrm{H}, \mathrm{CO}-\mathrm{CH}), 5.06(\mathrm{~s}, 1 \mathrm{H}$, Ar-OH), 4.17 (s, 1H, C-NH-C), 1.68 (s, 3H, C-CH3). ${ }^{13} \mathrm{C}$ NMR (400 $\left.\mathrm{MHz}, \mathrm{CDCl}_{3}, \delta, \mathrm{ppm}\right): 160.8$ (1C, $\left.>C=0\right), 152.2-110.3(14 \mathrm{C}, \mathrm{Ar}$ ), $21.4\left(-\mathrm{CH}_{3}\right.$ of $\alpha$-pyranone). MS (DIPMS, $\left.\mathrm{m} / \mathrm{z}\right): 296.04\left(\mathrm{M}^{+}\right)$and HMS calculated for $\mathrm{C}_{16} \mathrm{H}_{11} \mathrm{NO}_{3} \mathrm{~S}: 297.046$. Found: 297.0456.

10-chloro-4-methylpyrano[2,3-ß]phenothiazin-2(11H)-one (IIIe): Yield: 59.2\%. M.p.: 183-185 ${ }^{\circ} \mathrm{C}$. FT-IR $\left(\mathrm{KBr}, \mathrm{cm}^{-1}\right): 3446$ $v(\mathrm{~N}-\mathrm{H})$ (hetero), $3059 \quad v(\mathrm{C}-\mathrm{H})$ (aromatic), $2850 \quad v(\mathrm{C}-\mathrm{H})$ (aliphatic), $1749 v(\mathrm{C}=0)$ (pyraneone), $1571 v(\mathrm{C}-\mathrm{N})$ (hetero), $1450 v(\mathrm{C}=\mathrm{C})$ (aromatic), $1090 v(\mathrm{C}-\mathrm{Cl})$ (aromatic), $666 v(\mathrm{C}-\mathrm{S})$ (hetero). ${ }^{1} \mathrm{H}$ NMR $\left(400 \mathrm{MHz}, \mathrm{DMSO}-d_{6}, \delta, \mathrm{ppm}\right): 6.24-7.10(\mathrm{~m}$, $5 \mathrm{H}, \mathrm{Ar}-\mathrm{H}), 5.74(\mathrm{~s}, 1 \mathrm{H}, \mathrm{CO}-\mathrm{CH}), 4.15(\mathrm{~s}, 1 \mathrm{H}, \mathrm{C}-\mathrm{N} H-\mathrm{C}), 1.68(\mathrm{~s}, 3 \mathrm{H}$, $\left.\mathrm{C}-\mathrm{CH}_{3}\right) .{ }^{13} \mathrm{C}$ NMR $\left(400 \mathrm{MHz}, \mathrm{CDCl}_{3}, \delta, \mathrm{ppm}\right): 160.0(1 \mathrm{C},>C=0)$, 152.7-110.1 (14C, Ar-C), 21.7 (1C, $\left.-\mathrm{CH}_{3}\right)$. MS (DIPMS, m/z): $314.01\left(\mathrm{M}^{+}\right)$and $\mathrm{HMS}$ calculated for $\mathrm{C}_{16} \mathrm{H}_{10} \mathrm{ClNO}_{2} \mathrm{~S}$ : 314. 0121. Found: 314.0127.

9-chloro-4-methylpyrano[2,3-ß]phenothiazin-2(11H)-one (IIIf): Yield: $51.8 \%$. M.p.: $175-177$ ०C. FT-IR $\left(\mathrm{KBr}, \mathrm{cm}^{-1}\right): 3402 v(\mathrm{~N}-\mathrm{H})$ (hetero), $3057 v(\mathrm{C}-\mathrm{H})$ (aromatic), $2883 v(\mathrm{C}-\mathrm{H})$ (aliphatic), 1745 $v(\mathrm{C}=\mathrm{O})$ (pyraneone), $1595 \mathrm{v}(\mathrm{C}-\mathrm{N})$ (hetero), $1456 \mathrm{v}(\mathrm{C}=\mathrm{C})$ (aromatic), $1088 \mathrm{v}\left(\mathrm{C}-\mathrm{Cl}\right.$ ) (aromatic), $698 \mathrm{v}(\mathrm{C}-\mathrm{S})$ (hetero). ${ }^{1} \mathrm{H}$ NMR $\left(400 \mathrm{MHz}, \mathrm{DMSO}-d_{6}, \delta, \mathrm{ppm}\right): 6.56-6.93(\mathrm{~m}, 4 \mathrm{H}, \mathrm{Ar}-\mathrm{H})$, $6.06(\mathrm{~s}, 1 \mathrm{H}, \operatorname{Ar}-H), 5.81(\mathrm{~s}, 1 \mathrm{H}, \mathrm{CO}-\mathrm{CH}), 4.16(\mathrm{~s}, 1 \mathrm{H}, \mathrm{C}-\mathrm{N} H-\mathrm{C})$, $1.67\left(\mathrm{~s}, 3 \mathrm{H}, \mathrm{C}-\mathrm{CH}_{3}\right) .{ }^{13} \mathrm{C}$ NMR $\left(400 \mathrm{MHz}, \mathrm{CDCl}_{3}, \delta, \mathrm{ppm}\right): 160.5$ $(1 \mathrm{C},>\mathrm{C}=0), 152.2-110.9$ (14C, $\mathrm{Ar}-\mathrm{C}), 21.1\left(1 \mathrm{C},-\mathrm{CH}_{3}\right) . \mathrm{MS}$ (DIPMS, $\mathrm{m} / \mathrm{z}): 314.01\left(\mathrm{M}^{+}\right)$and HMS calculated for $\mathrm{C}_{16} \mathrm{H}_{10} \mathrm{ClNO}_{2} \mathrm{~S}: 314.0121$. Found: 314.0127.

8-chloro-4-methylpyrano[2,3-ß]phenothiazin-2(11H)-one (IIIg): Yield: $44.4 \%$. M.p.: $170-172{ }^{\circ} \mathrm{C}$. FT-IR $\left(\mathrm{KBr}, \mathrm{cm}^{-1}\right): 3363$ $v(\mathrm{~N}-\mathrm{H})$ (hetero), $3074 \quad v(\mathrm{C}-\mathrm{H})$ (aromatic), $2933 v(\mathrm{C}-\mathrm{H})$ (aliphatic), $1710 v(\mathrm{C}=0)$ (pyraneone), $1606 v(\mathrm{C}-\mathrm{N})$ (hetero), $1086 v(\mathrm{C}-\mathrm{Cl})$ (aromatic), $1415 v(\mathrm{C}=\mathrm{C})$ (aromatic), $705 v(\mathrm{C}-\mathrm{S})$ (hetero). ${ }^{1} \mathrm{H}$ NMR $\left(400 \mathrm{MHz}, \mathrm{DMSO}-d_{6}, \delta, \mathrm{ppm}\right): 7.02-7.07$ (m, $1 \mathrm{H}, \mathrm{Ar}-H), 6.82(\mathrm{~s}, 1 \mathrm{H}, \mathrm{Ar}-H), 6.61-6.63(\mathrm{~d}, J=7.6 \mathrm{~Hz}, 1 \mathrm{H}, \mathrm{Ar}-H)$, 6.34 (s, $1 \mathrm{H}, \mathrm{Ar}-H), 5.98(\mathrm{~s}, 1 \mathrm{H}, \mathrm{Ar}-H), 5.90$ (s, 1H, CO-CH), 4.20 (s, $1 \mathrm{H}, \mathrm{C}-\mathrm{NH}-\mathrm{C}), 1.65\left(\mathrm{~s}, 3 \mathrm{H}, \mathrm{C}-\mathrm{CH}_{3}\right) .{ }^{13} \mathrm{C}$ NMR $\left(400 \mathrm{MHz}, \mathrm{CDCl}_{3}, \delta\right.$, ppm): $161.9(1 \mathrm{C},>C=0), 152.6-110.1$ (14C, Ar-C), 21.3 (1C, $\mathrm{CH}_{3}$ ). MS (DIPMS, $\left.\mathrm{m} / \mathrm{z}\right): 314.01\left(\mathrm{M}^{+}\right)$and HMS calculated for $\mathrm{C}_{16} \mathrm{H}_{10} \mathrm{ClNO}_{2} \mathrm{~S}: 314.0121$. Found: 314.0127.

4-methyl-9-nitropyrano[2,3-ß]phenothiazin-2(11H)-one

(IIIh): Yield: 51\%. M.p.: 180-182 ${ }^{\circ} \mathrm{C}$. FT-IR $\left(\mathrm{KBr}, \mathrm{cm}^{-1}\right): 3318$ $v(\mathrm{~N}-\mathrm{H})$ (hetero), $3105 \quad v(\mathrm{C}-\mathrm{H})$ (aromatic), $2864 \quad v(\mathrm{C}-\mathrm{H})$ (aliphatic), $1719 v(\mathrm{C}=\mathrm{O})$ (pyraneone), $1552 v(\mathrm{C}-\mathrm{N})$ (Nitro aromatic), $1510 v(\mathrm{C}-\mathrm{N})$ (hetero), $1458 v(\mathrm{C}=\mathrm{C})$ (aromatic), 675 $v(\mathrm{C}-\mathrm{S})$ (hetero). ${ }^{1} \mathrm{H}$ NMR $\left(400 \mathrm{MHz}, \mathrm{DMSO}-d_{6}, \delta, \mathrm{ppm}\right): 7.73-7.80$ (dd, $J=8.8 \mathrm{~Hz}, 1 \mathrm{H}, \mathrm{Ar}-H$ ) , 7.42 (s, $1 \mathrm{H}, \mathrm{Ar}-H$ ) , 7.16-7.18 (d, $J=8 \mathrm{~Hz}$, $1 \mathrm{H}, \mathrm{Ar}-H), 6.76$ (s, 1H, Ar- $H$ ), 6.07 (s, 1H, Ar- $H$ ), 5.93 (s, 1H, CO$\mathrm{CH}), 4.18(\mathrm{~s}, 1 \mathrm{H}, \mathrm{C}-\mathrm{N} H-\mathrm{C}), 1.73\left(\mathrm{~s}, 3 \mathrm{H}, \mathrm{C}-\mathrm{CH}_{3}\right) .{ }^{13} \mathrm{C}$ NMR $(400$ $\left.\mathrm{MHz}, \mathrm{CDCl}_{3}, \delta, \mathrm{ppm}\right): 160.0(1 \mathrm{C},>C=0), 152.4-110.4(14 \mathrm{C}, \mathrm{Ar}-\mathrm{C})$, $21.7\left(1 \mathrm{C},-\mathrm{CH}_{3}\right)$. MS (DIPMS, m/z): $325.03\left(\mathrm{M}^{+}\right)$and HMS calculated for $\mathrm{C}_{16} \mathrm{H}_{10} \mathrm{~N}_{2} \mathrm{O}_{4} \mathrm{~S}: 326.0361$. Found: 326.0350 .

4-methyl-8-nitropyrano[2,3-3]phenothiazin-2(11H)-one (IIIi): Yield: 54.5\%. M.p.: $178-180^{\circ} \mathrm{C}$. FT-IR (KBr, cm$\left.{ }^{-1}\right): 3256$ $v(\mathrm{~N}-\mathrm{H})$ (hetero), $3072 \quad v(\mathrm{C}-\mathrm{H})$ (aromatic), $2866 \quad v(\mathrm{C}-\mathrm{H})$ (aliphatic), $1715 v(\mathrm{C}=0)$ (pyraneone), $1515 v(\mathrm{C}-\mathrm{N})$ (hetero), $1319 v(\mathrm{C}-\mathrm{N})$ (Nitro aromatic), $1461 v(\mathrm{C}=\mathrm{C})$ (aromatic), 675 $v(\mathrm{C}-\mathrm{S})$ (hetero). ${ }^{1} \mathrm{H}$ NMR $\left(400 \mathrm{MHz}\right.$, DMSO- $\left.d_{6}, \delta, \mathrm{ppm}\right): 7.80-7.87$ (dd, $J=8 \mathrm{~Hz}, 1 \mathrm{H}, \mathrm{Ar}-H$ ) , 6.91-6.92 (d, J=5.6 Hz, $1 \mathrm{H}, \mathrm{Ar}-H$ ), 7.64 (s, $1 \mathrm{H}, \mathrm{Ar}-H), 6.61(\mathrm{~s}, 1 \mathrm{H}, \mathrm{Ar}-H), 6.05$ (s, 1H, Ar- $H), 5.72$ (s, 1H, CO$\mathrm{CH}), 4.10(\mathrm{~s}, 1 \mathrm{H}, \mathrm{C}-\mathrm{N} H-\mathrm{C}), 1.68\left(\mathrm{~s}, 3 \mathrm{H}, \mathrm{C}-\mathrm{CH}_{3}\right) .{ }^{13} \mathrm{C}$ NMR $(400$ $\left.\mathrm{MHz}, \mathrm{CDCl}_{3}, \delta, \mathrm{ppm}\right): 160.3$ (1C, $\left.>\mathrm{C}=0\right), 153.4-110.9$ (14C, $\mathrm{Ar}-\mathrm{C}$ ), $21.1\left(1 \mathrm{C},-\mathrm{CH}_{3}\right)$. MS (DIPMS, m/z): $325.03\left(\mathrm{M}^{+}\right)$and HMS calculated for $\mathrm{C}_{16} \mathrm{H}_{10} \mathrm{~N}_{2} \mathrm{O}_{4} \mathrm{~S}: 326.0361$. Found: 326.0350 .

4-methyl-2-oxo-2, 11-dihydropyrano[2,3-ß]phenothiazine-8carboxylic acid (IIIj): Yield: 60\%. M.p.: 176-179 ${ }^{\circ} \mathrm{C}$. FT-IR (KBr, $\left.\mathrm{cm}^{-1}\right)$ : $3413 v(\mathrm{~N}-\mathrm{H})$ (hetero), $3035 \mathrm{v}(\mathrm{C}-\mathrm{H})$ (aromatic), $2923 \mathrm{v}(\mathrm{C}-$ H) (aliphatic), $1714 v(\mathrm{C}=0)$ (pyraneone), $1680 \vee(\mathrm{C}=0$ acid stretching), $1475 v(\mathrm{C}-\mathrm{N})$ (hetero) , $1427 \mathrm{v}(\mathrm{C}=\mathrm{C})$ (aromatic), 


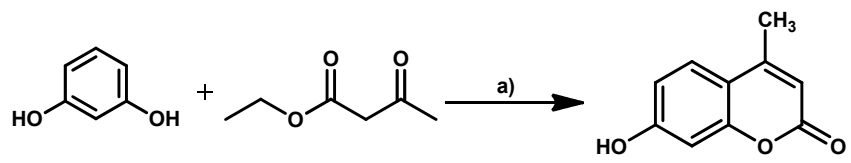

(I)<smiles>[R]c1ccc(N)cc1</smiles>

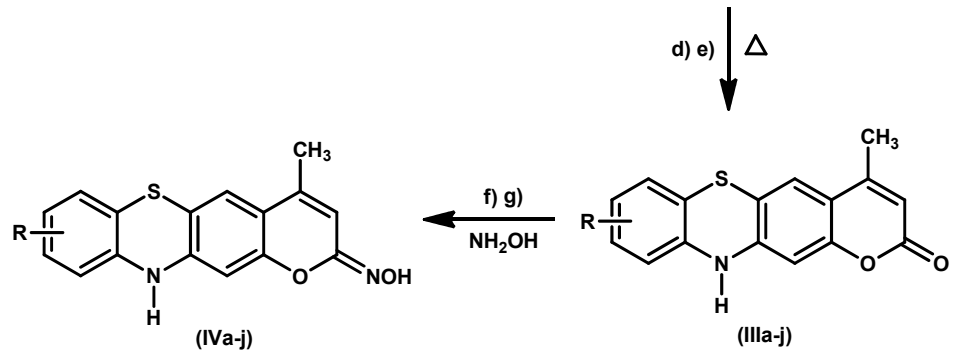

$\begin{array}{ll}\text { a, } \mathrm{R}=\mathrm{H} & \text { a) Concentrated } \mathrm{H}_{2} \mathrm{SO}_{4} \\ \text { b, } \mathrm{R}=2-\mathrm{OH} & \text { b) Absolute } \mathrm{C}_{2} \mathrm{H}_{5} \mathrm{OH} \\ \text { c, } \mathrm{R}=3-\mathrm{OH} & \text { c) Anhydrous } \mathrm{ZnCl}_{2} \\ \text { d, } \mathrm{R}=4-\mathrm{OH} & \text { d) Sulphur } \\ \text { e, } \mathrm{R}=2-\mathrm{Cl} & \text { e) lodine } \\ \text { f, R=3-Cl } & \text { f) } \mathrm{C}_{2} \mathrm{H}_{5} \mathrm{OH} \\ \text { g, } \mathrm{R}=4-\mathrm{Cl} & \text { g) Pyridine } \\ \text { h, R=3-NO } & \\ \text { i, R=4-NO } & \\ \text { j, R=4-COOH } & \end{array}$

Scheme 1

$1395 v(\mathrm{C}-\mathrm{O})$ (acid), $1271 v(\mathrm{O}-\mathrm{H})$ (acid), $690 v(\mathrm{C}-\mathrm{S})$ (hetero). ${ }^{1} \mathrm{H}$ NMR (400 MHz, DMSO- $\left.d_{6}, \delta, \mathrm{ppm}\right): 10.57$ (s, $\left.1 \mathrm{H}, \mathrm{O}-\mathrm{H}-\mathrm{COOH}\right)$, 7.87-7.92 (dd, J=8 Hz, 1H, Ar- H, ), 7.84 (s, $1 \mathrm{H}, \mathrm{Ar}-H), 6.91$ (s, $1 \mathrm{H}$, $\mathrm{Ar}-H), 6.89$ (s, 1H, Ar-H), 6.16 (s, 1H, Ar- $H$ ), 5.82 (s, 1H, CO-CH), 4.12 (s, 1H, C-NH-C), 1.71 (s, 3H, C-CH3). ${ }^{13} \mathrm{C}$ NMR $(400 \mathrm{MHz}$, $\left.\mathrm{CDCl}_{3}, \delta, \mathrm{ppm}\right): 160.2(1 \mathrm{C},>\mathrm{C}=0), 169.8(1 \mathrm{C}, \mathrm{Ar}=\mathrm{COOH}), 152.8-$ $110.1(14 \mathrm{C}, \mathrm{Ar}-\mathrm{C}), 21.3\left(1 \mathrm{C},-\mathrm{CH}_{3}\right)$. MS (DIPMS, m/z): 324.04 $\left(\mathrm{M}^{+}\right)$and HMS calculated for $\mathrm{C}_{17} \mathrm{H}_{11} \mathrm{NO}_{4} \mathrm{~S}$ : 325 . 0409. Found: 325.0452 .

\subsubsection{Preparation of compounds (IVa-j) [37,38]}

To a solution of the keto-ester $(1.44 \mathrm{~g}, 10 \mathrm{mmol})$ and benzyl-hydroxylamine $(1.60 \mathrm{~g}, 10 \mathrm{mmol})$ in ethanol $(30 \mathrm{~mL})$ was added pyridine $(5.0 \mathrm{~mL}, 62 \mathrm{mmol})$ in 1 portion. The reaction mixture was heated at $55{ }^{\circ} \mathrm{C}$ for $24 \mathrm{~h}$ and then concentrated on a rotary evaporator. The residue was partitioned between ether $(150 \mathrm{~mL})$ and water $(50 \mathrm{~mL})$. The organic layer was sequentially washed with hydrochloric acid $(0.5 \mathrm{~N}, 60 \mathrm{~mL})$ and water $(30 \mathrm{~mL})$, and then dried over Magnesium sulphate. Concentration in vacuo provided the oxime $(2.50 \mathrm{~g}, 100 \%)$ as a $3: 1$ mixture of $E Z$ isomers as a solids or liquid.

4-methylpyrano $2,3-\beta]$ phenothiazin-2(11H)-one oxime (IVa): Yield: $46.6 \%$. M.p.: $186-188{ }^{\circ} \mathrm{C}$. FT-IR $\left(\mathrm{KBr}, \mathrm{cm}^{-1}\right): 3396$ $v(\mathrm{~N}-\mathrm{H})$ (hetero), $3252 v(\mathrm{O}-\mathrm{H})$ (oxime), $3007 \mathrm{v}(\mathrm{C}-\mathrm{H})$ (aromatic), $2840 v(\mathrm{C}-\mathrm{H})$ (aliphatic), $1622 v(\mathrm{C}=\mathrm{N})$ (oxime), $1487 v(\mathrm{C}-\mathrm{N})$ (hetero), $1412 v(\mathrm{C}=\mathrm{C})$ (aromatic), $703 v(\mathrm{C}-\mathrm{S})$ (hetero). ${ }^{1} \mathrm{H}$ NMR
(400 MHz, DMSO- $\left.d_{6}, \delta, \mathrm{ppm}\right): 6.92(\mathrm{~s}, 1 \mathrm{H}, \mathrm{Ar}-H), 6.26-6.88$ (m, $4 \mathrm{H}, \mathrm{Ar}-H), 6.07$ (s, $1 \mathrm{H}, \mathrm{Ar}-H), 5.05(\mathrm{~s}, 1 \mathrm{H}, \mathrm{N}=\mathrm{C}-\mathrm{C}-H), 4.17(\mathrm{~s}, 1 \mathrm{H}$, C-NH-C), 2.07 (s, $1 \mathrm{H}, \mathrm{C}=\mathrm{N}-\mathrm{OH}), 1.68\left(\mathrm{~s}, 3 \mathrm{H}, \mathrm{C}-\mathrm{CH}_{3}{ }^{13} \mathrm{C}\right.$ NMR $(400$ $\left.\mathrm{MHz}, \mathrm{CDCl}_{3}, \delta, \mathrm{ppm}\right): 168.3(1 \mathrm{C},>\mathrm{C}=\mathrm{N}-\mathrm{OH}), 153.3-106.4(14 \mathrm{C}$, $\mathrm{Ar}-\mathrm{C}), 22.5\left(1 \mathrm{C},-\mathrm{CH}_{3}\right)$. MS (DIPMS, m/z): $295.06\left(\mathrm{M}^{+}\right)$and HMS calculated for $\mathrm{C}_{16} \mathrm{H}_{12} \mathrm{~N}_{2} \mathrm{O}_{2} \mathrm{~S}: 296.061$. Found: 296.0609 .

10-hydroxy-4-methylpyrano[2,3-ß]phenothiazin-2(11H)-one oxime (IVb): Yield: $45.5 \%$. M.p.: $184-186^{\circ} \mathrm{C}$. FT-IR $\left(\mathrm{KBr}, \mathrm{cm}^{-1}\right)$ : $3429 v(\mathrm{O}-\mathrm{H})$ (phenol), $3419 \mathrm{v}(\mathrm{N}-\mathrm{H})$ (hetero), $3315 \mathrm{v}(\mathrm{O}-\mathrm{H})$ (oxime), $3053 v(\mathrm{C}-\mathrm{H})$ (aromatic), $2885 \mathrm{v}(\mathrm{C}-\mathrm{H})$ (aliphatic), 1656 $v(\mathrm{C}=\mathrm{N})$ (oxime), $1515 v(\mathrm{C}-\mathrm{N})$ (hetero), $1450 \vee(\mathrm{C}=\mathrm{C})$ (aromatic), $1211 \mathrm{v}(\mathrm{C}-0)$ (aromatic), $696 \mathrm{v}(\mathrm{C}-\mathrm{S})$ (hetero). ${ }^{1} \mathrm{H}$ NMR $(400$ MHz, DMSO- $\left.d_{6}, \delta, p p m\right): 6.30-6.72(\mathrm{~m}, 5 \mathrm{H}, \mathrm{Ar}-H), 5.18(\mathrm{~s}, 1 \mathrm{H}$, $\mathrm{N}=\mathrm{C}-\mathrm{C}-H), 5.00(\mathrm{~s}, 1 \mathrm{H}, \mathrm{Ar}-\mathrm{OH}), 4.13(\mathrm{~s}, 1 \mathrm{H}, \mathrm{C}-\mathrm{N} H-\mathrm{C}), 2.03(\mathrm{~s}, 1 \mathrm{H}$, $\mathrm{C}=\mathrm{N}-\mathrm{OH}), 1.78\left(\mathrm{~s}, 3 \mathrm{H}, \mathrm{C}-\mathrm{CH}_{3}\right) .{ }^{13} \mathrm{C}$ NMR $\left(400 \mathrm{MHz}, \mathrm{CDCl}_{3}, \delta\right.$, ppm): 168.1 (1C, >C=N-OH), 153.1-105.8 (14C, Ar-C), 22.3 (1C, $\mathrm{CH}_{3}$ ). MS (DIPMS, $\mathrm{m} / \mathrm{z}$ ): $311.05\left(\mathrm{M}^{+}\right)$and HMS calculated for $\mathrm{C}_{16} \mathrm{H}_{12} \mathrm{~N}_{2} \mathrm{O}_{3} \mathrm{~S}$ : 312.056. Found: 312.0550 .

9-hydroxy-4-methylpyrano[2,3-ß]phenothiazin-2(11H)-one oxime (IVc): Yield: $45.4 \%$. M.p.: $194-196{ }^{\circ} \mathrm{C}$. FT-IR $\left(\mathrm{KBr}, \mathrm{cm}^{-1}\right)$ : $3384 v(\mathrm{~N}-\mathrm{H})$ (hetero) , $3332 \mathrm{v}(\mathrm{O}-\mathrm{H})$ (phenol), $3211 \mathrm{v}(\mathrm{O}-\mathrm{H})$ (oxime), $3053 v(\mathrm{C}-\mathrm{H})$ (aromatic), $2889 v(\mathrm{C}-\mathrm{H})$ (aliphatic), 1637 $v(\mathrm{C}=\mathrm{N})$ (oxime), $1533 v(\mathrm{C}-\mathrm{N})$ (hetero), $1438 v(\mathrm{C}=\mathrm{C})$ (aromatic), $1220 v(\mathrm{C}-0)$ (aromatic), $665 \mathrm{v}(\mathrm{C}-\mathrm{S})$ (hetero). ${ }^{1} \mathrm{H}$ NMR $(400$ MHz, DMSO- $\left.d_{6}, \delta, \mathrm{ppm}\right): 6.15-6.69(\mathrm{~m}, 5 \mathrm{H}, \mathrm{Ar}-H), 5.19(\mathrm{~s}, 1 \mathrm{H}$ $\mathrm{N}=\mathrm{C}-\mathrm{C}-H), 5.06(\mathrm{~s}, 1 \mathrm{H}, \mathrm{Ar}-\mathrm{OH}), 4.17(\mathrm{~s}, 1 \mathrm{H}, \mathrm{C}-\mathrm{N} H-\mathrm{C}), 2.04(\mathrm{~s}, 1 \mathrm{H}$, $\mathrm{C}=\mathrm{N}-\mathrm{OH}), 1.07\left(\mathrm{~s}, 3 \mathrm{H}, \mathrm{C}-\mathrm{CH}_{3}\right) \cdot{ }^{13} \mathrm{C}$ NMR $\left(400 \mathrm{MHz}, \mathrm{CDCl}_{3}, \delta\right.$, ppm): $168.5(1 \mathrm{C},>\mathrm{C}=\mathrm{N}-\mathrm{OH}), 154.0-103.7$ (14C, $\mathrm{Ar}-\mathrm{C}), 22.6$ (1C, - 
$\mathrm{CH}_{3}$ ). MS (DIPMS, m/z): $311.05\left(\mathrm{M}^{+}\right)$and HMS calculated for $\mathrm{C}_{16} \mathrm{H}_{12} \mathrm{~N}_{2} \mathrm{O}_{3} \mathrm{~S}: 312.056$. Found: 312.0550 .

8-hydroxy-4-methylpyrano[2,3-ß]phenothiazin-2(11H)-one oxime (IVd): Yield: $43.6 \%$. M.p.: $178-180{ }^{\circ} \mathrm{C}$. FT-IR $\left(\mathrm{KBr}, \mathrm{cm}^{-1}\right)$ : $3373 v(\mathrm{O}-\mathrm{H})$ (phenol), $3222 \mathrm{v}(\mathrm{N}-\mathrm{H})$ (hetero), $3101 \mathrm{v}(\mathrm{O}-\mathrm{H})$ (oxime), $3029 v(\mathrm{C}-\mathrm{H})$ (aromatic), $2862 v(\mathrm{C}-\mathrm{H})$ (aliphatic), 1631 $v(\mathrm{C}=\mathrm{N})$ (oxime), $1520 \quad v(\mathrm{C}-\mathrm{N})$ (hetero) , $1415 \quad v(\mathrm{C}=\mathrm{C})$ (aromatic), $1253 v(\mathrm{C}-\mathrm{O})$ (aromatic), $700 v\left(\mathrm{C}-\mathrm{S}\right.$ ) (hetero). ${ }^{1} \mathrm{H}$ NMR (400 MHz, DMSO- $\left.d_{6}, \delta, p p m\right): 6.13-6.71(\mathrm{~m}, 5 \mathrm{H}, \mathrm{Ar}-H)$, $5.18(\mathrm{~s}, 1 \mathrm{H}, \mathrm{N}=\mathrm{C}-\mathrm{C}-H), 5.06$ (s, $1 \mathrm{H}, \mathrm{Ar}-\mathrm{O} H), 4.17$ (s, $1 \mathrm{H}, \mathrm{C}-\mathrm{N} H-\mathrm{C}$ ), $2.10(\mathrm{~s}, 1 \mathrm{H}, \mathrm{C}=\mathrm{N}-\mathrm{OH}), 1.68\left(\mathrm{~s}, 3 \mathrm{H}, \mathrm{C}-\mathrm{CH}_{3}\right) .{ }^{13} \mathrm{C}$ NMR $(400 \mathrm{MHz}$, $\left.\mathrm{CDCl}_{3}, \delta, \mathrm{ppm}\right): 167.4(1 \mathrm{C},>C=\mathrm{N}-\mathrm{OH}), 152.5-106.2(14 \mathrm{C}, \mathrm{Ar}-\mathrm{C}$ ), $21.3\left(1 \mathrm{C},-\mathrm{CH}_{3}\right)$. MS (DIPMS, $\mathrm{m} / \mathrm{z}$ ): $311.05\left(\mathrm{M}^{+}\right)$and HMS calculated for $\mathrm{C}_{16} \mathrm{H}_{12} \mathrm{~N}_{2} \mathrm{O}_{3} \mathrm{~S}$ : 312.056. Found: 312.0550 .

10-chloro-4-methylpyrano[2,3-3]phenothiazin-2(11H)-one oxime (IVe): Yield: $43 \%$. M.p.: $192-194{ }^{\circ} \mathrm{C}$. FT-IR $\left(\mathrm{KBr}, \mathrm{cm}^{-1}\right)$ : $3338 v(\mathrm{~N}-\mathrm{H})$ (hetero), $3255 \mathrm{v}(\mathrm{O}-\mathrm{H})$ (oxime), $3049 v(\mathrm{C}-\mathrm{H})$ (aromatic), $2920 v(\mathrm{C}-\mathrm{H})$ (aliphatic), $1653 v(\mathrm{C}=\mathrm{N})$ (oxime), 1591 $v(\mathrm{C}-\mathrm{N})$ (hetero), $1499 v(\mathrm{C}=\mathrm{C})$ (aromatic), $1088 v(\mathrm{C}-\mathrm{Cl})$ (aromatic), $686 v(\mathrm{C}-\mathrm{S})$ (hetero). ${ }^{1} \mathrm{H}$ NMR $\left(400 \mathrm{MHz}, \mathrm{DMSO}-d_{6}, \delta\right.$, ppm): 7.00-7.07 (m, 1H, Ar- $H$ ), 6.82-6.84 (d, J=8 Hz, 1H, Ar- $H$ ), 6.61-6.64 (t, $J=6 \mathrm{~Hz}, 1 \mathrm{H}, \mathrm{Ar}-H), 6.74(\mathrm{~s}, 1 \mathrm{H}, \mathrm{Ar}-H), 6.06(\mathrm{~s}, 1 \mathrm{H}$, Ar- $H$ ), 5.10 (s, $1 \mathrm{H}, \mathrm{N}=\mathrm{C}-\mathrm{C}-H$ ), 4.15 (s, $1 \mathrm{H}, \mathrm{C}-\mathrm{N} H-\mathrm{C}), 1.97$ (s, $1 \mathrm{H}$, $\mathrm{C}=\mathrm{N}-\mathrm{OH}), 1.68\left(\mathrm{~s}, 3 \mathrm{H}, \mathrm{C}-\mathrm{CH}_{3}\right) \cdot{ }^{13} \mathrm{C}$ NMR $\left(400 \mathrm{MHz}, \mathrm{CDCl}_{3}, \delta\right.$, ppm): 166.2 (1C, >C=N-OH), 153.6-104.3 (14C, Ar-C), 22.7 ($\mathrm{CH}_{3}$ ). MS (DIPMS, $\mathrm{m} / \mathrm{z}$ ): $329.02\left(\mathrm{M}^{+}\right)$and HMS calculated for $\mathrm{C}_{16} \mathrm{H}_{11} \mathrm{ClN}_{2} \mathrm{O}_{2} \mathrm{~S}$ : 330.023. Found: 330.0220 .

9-chloro-4-methylpyrano[2,3-ß]phenothiazin-2(11H)-one oxime (IVf): Yield: $45.8 \%$. M.p.: $198-200{ }^{\circ} \mathrm{C}$. FT-IR $\left(\mathrm{KBr}, \mathrm{cm}^{-1}\right)$ : $3427 v(\mathrm{~N}-\mathrm{H})$ (hetero) , $3152 v(\mathrm{O}-\mathrm{H})$ (oxime), $3028 v(\mathrm{C}-\mathrm{H})$ (aromatic), $2881 v(\mathrm{C}-\mathrm{H})$ (aliphatic), $1618 v(\mathrm{C}=\mathrm{N})$ (oxime), 1500 $v(\mathrm{C}-\mathrm{N})$ (hetero), $1402 v(\mathrm{C}=\mathrm{C})$ (aromatic), $1098 v(\mathrm{C}-\mathrm{Cl})$ (aromatic), $703 v(\mathrm{C}-\mathrm{S})$ (hetero). ${ }^{1} \mathrm{H}$ NMR $\left(400 \mathrm{MHz}, \mathrm{DMSO}-d_{6}, \delta\right.$, ppm): 6.56-6.93 (m, 4H, Ar-H), $6.06(\mathrm{~s}, 1 \mathrm{H}, \mathrm{Ar}-H), 5.08$ (s, $1 \mathrm{H}$, $\mathrm{N}=\mathrm{C}-\mathrm{C}-H), 4.16(\mathrm{~s}, 1 \mathrm{H}, \mathrm{C}-\mathrm{N} H-\mathrm{C}), 1.93(\mathrm{~s}, 1 \mathrm{H}, \mathrm{C}=\mathrm{N}-\mathrm{OH}) 1.67$ (s, $\left.3 \mathrm{H}, \mathrm{C}-\mathrm{CH}_{3}\right) \cdot{ }^{13} \mathrm{C}$ NMR $\left(400 \mathrm{MHz}, \mathrm{CDCl}_{3}, \delta, \mathrm{ppm}\right): 168.0(1 \mathrm{C}$, $>C=\mathrm{N}-\mathrm{OH}), 152.3-105.4(14 \mathrm{C}, \mathrm{Ar}-\mathrm{C}), 22.2\left(1 \mathrm{C},-\mathrm{CH}_{3}\right)$. MS (DIPMS, $\mathrm{m} / \mathrm{z}): 329.02\left(\mathrm{M}^{+}\right)$and $\mathrm{HMS}$ calculated for $\mathrm{C}_{16} \mathrm{H}_{11} \mathrm{ClN}_{2} \mathrm{O}_{2} \mathrm{~S}$ : 330.023. Found: 330.0220 .

8-chloro-4-methylpyrano[2,3- $\beta$ ]phenothiazin-2(11H)-one oxime (IVg): Yield: $48.6 \%$. M.p.: $176-178{ }^{\circ} \mathrm{C}$. FT-IR $\left(\mathrm{KBr}, \mathrm{cm}^{-1}\right)$ : $3220 v(\mathrm{~N}-\mathrm{H})$ (hetero) , $3154 \mathrm{v}(\mathrm{O}-\mathrm{H})$ (oxime), $3020 \mathrm{v}(\mathrm{C}-\mathrm{H})$ (aromatic), $2957 v(\mathrm{C}-\mathrm{H})$ (aliphatic), $1625 \mathrm{v}(\mathrm{C}=\mathrm{N})$ (oxime), 1566 $v(\mathrm{C}-\mathrm{N})$ (hetero) , $1400 v(\mathrm{C}=\mathrm{C})$ (aromatic), $1094 v(\mathrm{C}-\mathrm{Cl})$ (aromatic), $692 v(\mathrm{C}-\mathrm{S})$ (hetero). ${ }^{1} \mathrm{H}$ NMR $\left(400 \mathrm{MHz}, \mathrm{DMSO}-d_{6}, \delta\right.$, ppm): 7.02-7.07 (m, 1H, Ar- $H$ ), $6.82(\mathrm{~s}, 1 \mathrm{H}, \mathrm{Ar}-H), 6.61-6.63$ (d, $\mathrm{J}=7.6 \mathrm{~Hz}, 1 \mathrm{H}, \mathrm{Ar}-\mathrm{H}$, ) $, 6.34(\mathrm{~s}, 1 \mathrm{H}, \mathrm{Ar}-H), 5.98$ (s, $1 \mathrm{H}, \mathrm{Ar}-\mathrm{H}), 5.17$ (s, 1H, N=C-C-H), 4.20 (s, 1H, C-NH-C), 1.97 (s, $1 \mathrm{H}, \mathrm{C}=\mathrm{N}-\mathrm{OH})$, $1.65\left(\mathrm{~s}, 3 \mathrm{H}, \mathrm{C}-\mathrm{CH}_{3}\right) .{ }^{13} \mathrm{C}$ NMR $\left(400 \mathrm{MHz}, \mathrm{CDCl}_{3}, \delta, \mathrm{ppm}\right): 168.9$ (1C, >C=N-OH), 151.6-106.0 (14C, Ar-C), 22.9 (1C, $\left.-\mathrm{CH}_{3}\right) . \mathrm{MS}$ (DIPMS, $\mathrm{m} / \mathrm{z}$ ): $329.02\left(\mathrm{M}^{+}\right)$and HMS calculated for $\mathrm{C}_{16} \mathrm{H}_{11} \mathrm{ClN}_{2} \mathrm{O}_{2} \mathrm{~S}$ : 330.023 . Found: 330.0220 .

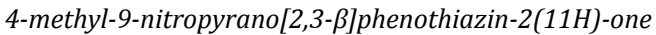
oxime (IVh): Yield: 40\%. M.p.: $196-198{ }^{\circ} \mathrm{C}$. FT-IR $\left(\mathrm{KBr}, \mathrm{cm}^{-1}\right)$ : $3228 v(\mathrm{~N}-\mathrm{H})$ (hetero) , $3145 \mathrm{v}(\mathrm{O}-\mathrm{H})$ (oxime), $3045 \mathrm{v}(\mathrm{C}-\mathrm{H})$ (aromatic), $2891 v(\mathrm{C}-\mathrm{H})$ (aliphatic), $1633 v(\mathrm{C}=\mathrm{N})$ (oxime), 1542 $v(\mathrm{C}-\mathrm{N})$ (nitro aromatic asym.), $1479 v(\mathrm{C}-\mathrm{N})$ (hetero), 1427 $v(\mathrm{C}=\mathrm{C})$ (aromatic), 1338 (C-N) (nitro aromatic sym.), $898 v(\mathrm{C}-$ $\mathrm{N}), 671 v(\mathrm{C}-\mathrm{S})$ (hetero). ${ }^{1} \mathrm{H}$ NMR $\left(400 \mathrm{MHz}, \mathrm{DMSO}-d_{6}, \delta, \mathrm{ppm}\right):$ 7.73-7.80 (dd, $J=8.8 \mathrm{~Hz}, 1 \mathrm{H}, \mathrm{Ar}-H$ ), 7.42 (s, $1 \mathrm{H}, \mathrm{Ar}-H$ ), 7.16-7.18 (d, J=8 Hz, 1H, Ar-H), 6.76 (s, 1H, Ar-H), 6.07 (s, 1H, Ar- H), 5.10 $(\mathrm{s}, 1 \mathrm{H}, \mathrm{N}=\mathrm{C}-\mathrm{C}-\mathrm{H}), 1.98(\mathrm{~s}, 1 \mathrm{H}, \mathrm{C}=\mathrm{N}-\mathrm{OH}), 1.73\left(\mathrm{~s}, 3 \mathrm{H}, \mathrm{C}-\mathrm{CH}_{3}\right), 4.18$ (s, $1 \mathrm{H}, \mathrm{C}-\mathrm{NH}-\mathrm{C}) .{ }^{13} \mathrm{C}$ NMR $\left(400 \mathrm{MHz}, \mathrm{CDCl}_{3}, \delta, \mathrm{ppm}\right): 168.7$ (1C, $>C=\mathrm{N}-\mathrm{OH}$ ), 152.4-105.0 (14C, $\mathrm{Ar}-\mathrm{C}$ ), $22.7\left(1 \mathrm{C},-\mathrm{CH}_{3}\right)$. MS (DIPMS, $\mathrm{m} / \mathrm{z}): 340.04\left(\mathrm{M}^{+}\right)$and HMS calculated for $\mathrm{C}_{16} \mathrm{H}_{11} \mathrm{~N}_{3} \mathrm{O}_{4} \mathrm{~S}$ : 341.047. Found: 341.0460 .

4-methyl-8-nitropyrano[2,3-ß]phenothiazin-2(11H)-one oxime (IVi): Yield: $45.2 \%$. M.p.: $166-168{ }^{\circ} \mathrm{C}$. FT-IR $\left(\mathrm{KBr}, \mathrm{cm}^{-1}\right)$ :
$3218 v(\mathrm{~N}-\mathrm{H})$ (hetero), $3154 v(\mathrm{O}-\mathrm{H})$ (oxime), $3031 v(\mathrm{C}-\mathrm{H})$ (aromatic), $2842 \quad v(\mathrm{C}-\mathrm{H})$ (aliphatic), $1660 \quad v(\mathrm{C}-\mathrm{N})$ (nitro aromatic asym.), $1645 \mathrm{v}(\mathrm{C}=\mathrm{N})$ (oxime), $1600 v(\mathrm{C}-\mathrm{N})$ (hetero), $1406 v(\mathrm{C}=\mathrm{C})$ (aromatic), $1338 v(\mathrm{C}-\mathrm{N})$ (nitro aromatic sym.) $860 v(\mathrm{C}-\mathrm{N}), 673 v(\mathrm{C}-\mathrm{S})$ (hetero). ${ }^{1} \mathrm{H}$ NMR $\left(400 \mathrm{MHz}, \mathrm{DMSO}-d_{6}, \delta\right.$, ppm): 7.80-7.87 (dd, $J=8 \mathrm{~Hz}, 1 \mathrm{H}, \mathrm{Ar}-H$ ), $7.64(\mathrm{~s}, 1 \mathrm{H}, \mathrm{Ar}-H$ ), 6.916.92 (d, J=5.6 Hz, 1H, Ar- $H$ ), 6.61 (s, 1H, Ar- $H$ ), 6.05 (s, 1H, Ar$H), 5.19(\mathrm{~s}, 1 \mathrm{H}, \mathrm{N}=\mathrm{C}-\mathrm{C}-\mathrm{H}), 4.10(\mathrm{~s}, 1 \mathrm{H}, \mathrm{C}-\mathrm{N} H-\mathrm{C}), 2.08(\mathrm{~s}, 1 \mathrm{H}, \mathrm{C}=$ $\mathrm{N}-\mathrm{OH}), 1.68\left(\mathrm{~s}, 3 \mathrm{H}, \mathrm{C}-\mathrm{CH}_{3}\right) .{ }^{13} \mathrm{C} \mathrm{NMR}\left(400 \mathrm{MHz}, \mathrm{CDCl}_{3}, \delta, \mathrm{ppm}\right)$ : $168.3(1 \mathrm{C},>C=\mathrm{N}-\mathrm{OH}), 155.2-103.8(14 \mathrm{C}, \mathrm{Ar}-\mathrm{C}), 22.1\left(1 \mathrm{C},-\mathrm{CH}_{3}\right)$. MS (DIPMS, m/z): $340.04\left(\mathrm{M}^{+}\right)$and HMS calculated for $\mathrm{C}_{16} \mathrm{H}_{11} \mathrm{~N}_{3} \mathrm{O}_{4} \mathrm{~S}$ : 341.047. Found: 341.0460 .

2-(hydroxyimino)-4-methyl-2,11-dihydropyrano[2, 3]phenothiazine-8-carboxylic acid (IVj): Yield: 48.3\%. M.p.: 168$170{ }^{\circ} \mathrm{C}$. FT-IR $\left(\mathrm{KBr}, \mathrm{cm}^{-1}\right): 3263 \mathrm{v}(\mathrm{N}-\mathrm{H})$ (hetero) , $3252 \mathrm{v}(\mathrm{O}-\mathrm{H})$ (oxime), $3053 v(\mathrm{C}-\mathrm{H})$ (aromatic), $2894 \mathrm{v}(\mathrm{C}-\mathrm{H})$ (aliphatic), 1685 $v(\mathrm{C}=0)$ (acid), $1622 v(\mathrm{C}=\mathrm{N})$ (oxime), $1587 v(\mathrm{C}-\mathrm{N})$ (hetero), $1450 v(\mathrm{C}-\mathrm{O})$ (acid), $1402 \vee(\mathrm{C}=\mathrm{C})$ (aromatic), $1319 v(\mathrm{O}-\mathrm{H})$ (acid), $689 v(\mathrm{C}-\mathrm{S})$ (hetero). ${ }^{1} \mathrm{H}$ NMR $\left(400 \mathrm{MHz}, \mathrm{DMSO}-d_{6}, \delta\right.$, ppm): 10.57 (s, $1 \mathrm{H}, \mathrm{Ar}-\mathrm{COOH}$ ), 7.87-7.92 (dd, $J=8 \mathrm{~Hz}, 1 \mathrm{H}, \mathrm{Ar}-\mathrm{H}$ ), $6.91(\mathrm{~s}, 1 \mathrm{H}, \operatorname{Ar}-H), 7.84(\mathrm{~s}, 1 \mathrm{H}, \operatorname{Ar}-H), 6.89$ (s, IH, Ar- $H$ ), 6.00 (s, $1 \mathrm{H}, \mathrm{Ar}-H), 5.06$ (s, 1H, N=C-C-H), 4.12 (s, $1 \mathrm{H}, \mathrm{C}-\mathrm{N} H-\mathrm{C}), 2.00$ (s, $1 \mathrm{H}, \mathrm{C}=\mathrm{N}-\mathrm{OH}), 1.71\left(\mathrm{~s}, 3 \mathrm{H}, \mathrm{C}-\mathrm{CH}_{3}\right) \cdot{ }^{13} \mathrm{C}$ NMR $\left(400 \mathrm{MHz}, \mathrm{CDCl}_{3}, \delta\right.$, ppm): $168.2(1 \mathrm{C},>\mathrm{C}=\mathrm{N}-\mathrm{OH}), 169.8(1 \mathrm{C},=\mathrm{COOH}), 155.8-105.7$ (14C, $\mathrm{Ar}-\mathrm{C}$ ), $22.3\left(1 \mathrm{C},-\mathrm{CH}_{3}\right)$. MS (DIPMS, $\left.\mathrm{m} / \mathrm{z}\right): 339.05\left(\mathrm{M}^{+}\right)$and HMS calculated for $\mathrm{C}_{17} \mathrm{H}_{12} \mathrm{~N}_{2} \mathrm{O}_{4} \mathrm{~S}: 340.051$. Found: 340.0528 .

\subsection{Biological activity}

The synthesized compounds were screened for the antibacterial and antifungal activity by using the agar-cup technique in nutrients agar and potato dextrose agar media, respectively [39-50]. Ciprofloxacin and gresiofluvin were used as standard drug for the antibacterial and antifungal activity respectively and zone of inhibition of all newly synthesized compounds (IIIa-j) and (IVa-j) was measured against these standard drugs (Table 1 and 2). The novel synthesized compounds have shown moderate activity against bacterial strain compared to standard drug. The title compounds have showed the better antibacterial activity and antifungal activity compared with the standard drug. We used microbial strainsStaphylococcus aureus (NCIM 2602); Bacillus subtilis (NCIM 2613) as Gr(+) bacterial strains, Escherichia coli (NCIM 2666); Pseudomonas aeruginosa (NCIM 5225) Gr (-) bacterial strains as Saccharomyces cerevisiae (NCIM 3220); Candida albicans (NCIM 3471); Aspergillus niger (NCIM 813) as fungal strains for biological test.

\section{Result and discussion}

We have synthesized a series of ten novel 4-methyl-2-oxopyranyl-phenothiazines (IIIa-j) derivatives and their oxime derivatives i.e. 4-methylpyrano-(2,3- $\beta$ )-phenothiazine-2(11H)one oxime (IVa-j) using 7-hydroxy-4methyl-coumarin. Structures of the synthesized compounds were established on the basis of IR, ${ }^{1} \mathrm{H}$ NMR, Mass and HMS spectral data in order to substantiate the structures of the compounds.

Compounds (IIIa-j) have showed presence of absorption band at $670-715 \mathrm{~cm}^{-1}$ conformed the formation of phenothiazine ring (C-S hetero stretching), sharp bands at $1715-1775 \mathrm{~cm}^{-1}$ gave conformation of $>\mathrm{C}=0$ in $\alpha$-pyrone ring in their respective spectra. Furthermore compounds (IVa-j) have showed presence of absorption band ranging from 1620-1690 $\mathrm{cm}^{-1}$ for oxime ( $\mathrm{C}=\mathrm{N}$ ) (oxime), and also showed presence of absorption band ranging from $3150-3300 \mathrm{~cm}^{-1}$ for oxime $(\mathrm{O}-\mathrm{H})$ (oxime) gave conformation of formation of oxime $(>\mathrm{C}=\mathrm{N}-\mathrm{OH})$ oxime group in their respective spectra. 
Table 1. Antibacterial and antifungal activity of synthesized novel series of 4-methyl-2-oxo-pyranyl-phenothiazines (IIIa-j) derivatives by cup-plate (agar cup) method*.

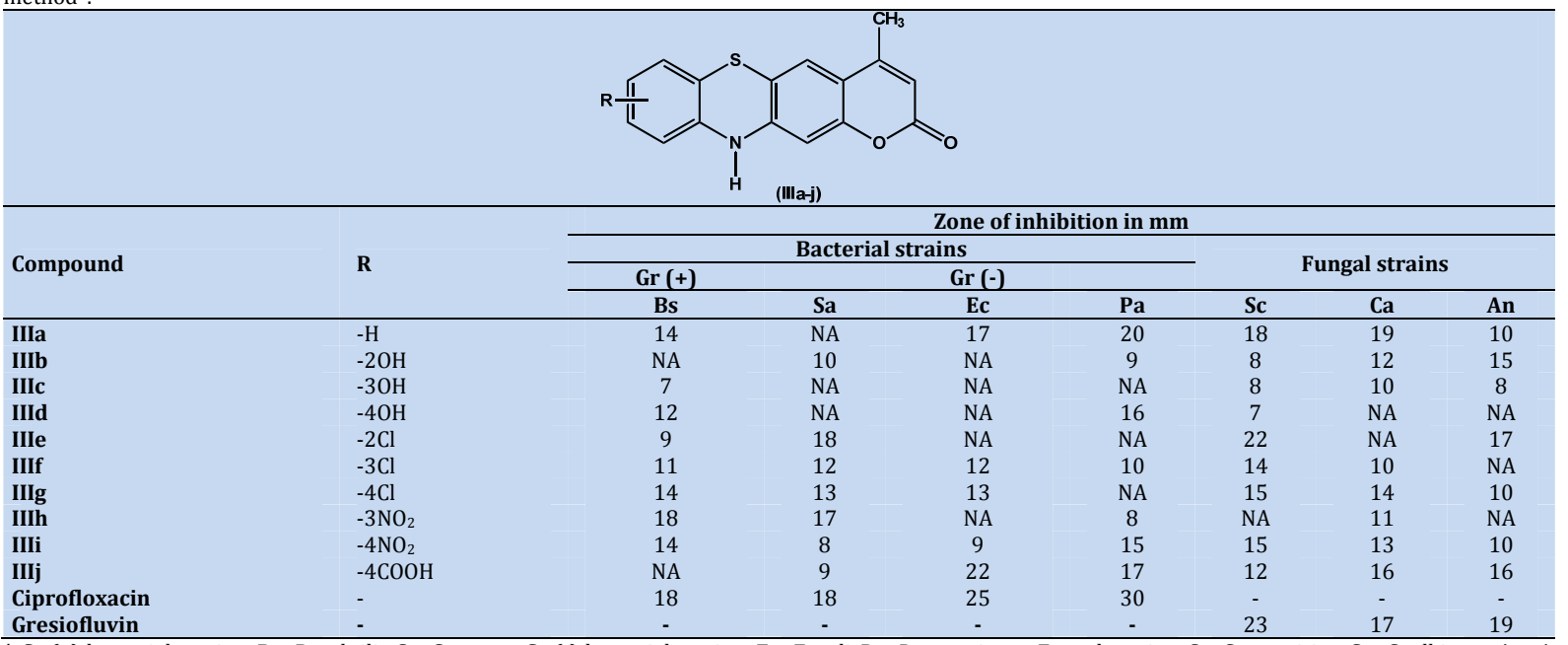

* Gr (+) bacterial strains: Bs: B. subtilis, Sa: S. aureus Gr (-) bacterial strains: Ec: E. coli, Pa: P. aeruginosa, Fungal strains: Sc: S. cerevisiae, Ca: C. albicans, An: A. niger. The concentration of test compounds were $100 \mu \mathrm{g} / \mathrm{mL}$. Solvent used DMF. NA = Not active.

Table 2. Antibacterial and antifungal activity of synthesized novel series of 4-methylpyrano (2,3- $\beta$ ) phenothiazine-2(11H)-one oxime (IVa-j) derivatives by cupplate (agar cup) method.

\begin{tabular}{|c|c|c|c|c|c|c|c|c|}
\hline \multirow{4}{*}{ Compound } & \multirow{4}{*}{$\mathbf{R}$} & \multicolumn{7}{|c|}{ Zone of inhibition in $\mathrm{mm}$} \\
\hline & & \multicolumn{4}{|c|}{ Bacterial strains } & \multirow{2}{*}{\multicolumn{3}{|c|}{ Fungal strains }} \\
\hline & & $\operatorname{Gr}(+)$ & & Gr (- & & & & \\
\hline & & Bs & Sa & Ec & $\mathbf{P a}$ & Sc & $\mathbf{C a}$ & $\mathbf{A n}$ \\
\hline IVa & $-\mathrm{H}$ & 14 & 18 & 23 & 14 & 27 & 15 & NA \\
\hline IVb & $-2 \mathrm{OH}$ & NA & 10 & NA & NA & 8 & 12 & 10 \\
\hline IVc & $-30 \mathrm{H}$ & 9 & NA & 8 & 15 & NA & 11 & 13 \\
\hline IVd & $-4 \mathrm{OH}$ & NA & 18 & 11 & NA & NA & 12 & 14 \\
\hline IVe & $-2 \mathrm{Cl}$ & 11 & NA & NA & 10 & 16 & 14 & NA \\
\hline IVf & $-3 \mathrm{Cl}$ & 12 & 13 & NA & NA & 12 & 10 & 8 \\
\hline IVg & $-4 \mathrm{Cl}$ & 11 & NA & NA & 13 & 11 & 14 & NA \\
\hline IVh & $-3 \mathrm{NO}_{2}$ & 13 & 12 & NA & 18 & NA & 17 & 10 \\
\hline IVi & $-4 \mathrm{NO}_{2}$ & 10 & 10 & NA & 12 & 7 & 10 & 16 \\
\hline IVj & $-4 \mathrm{COOH}$ & 15 & 17 & 10 & 19 & 10 & 14 & 15 \\
\hline Ciprofloxacin & - & 18 & 18 & 25 & 30 & - & - & - \\
\hline Gresiofluvin & - & - & - & - & & 23 & 17 & 18 \\
\hline
\end{tabular}

* Gr (+) bacterial strains: Bs: B. subtilis, Sa: S. aureus Gr (-) bacterial strains: Ec: E. coli, Pa: P. aeruginosa, Fungal strains: Sc: S. cerevisiae, Ca: C. albicans, An: A. niger. The concentration of test compounds were $100 \mu \mathrm{g} / \mathrm{mL}$. Solvent used DMF. NA = Not active.

In particular, it must be pointed out that in ${ }^{1} \mathrm{H}$ NMR the characteristic peaks at $\delta 1.70 \mathrm{ppm}\left(\mathrm{CH}_{3}\right.$ group of $\alpha$-pyrone ring), $\delta 4.17 \mathrm{ppm}$ (N-H group of phenothiazine), $\delta 5.67 \mathrm{ppm}(\mathrm{C}-$ H-Pyran), 6.26 (C-H-Ar) and a multiplate at $\delta 6.30-6.88 \mathrm{ppm}$ $(4 \mathrm{H}, \mathrm{C}-\mathrm{H}-\mathrm{Ar})$ indicate the presence of above groups in their respective structure.

The compounds (IIIa-j) showed prominent singlet at $\delta$ 6.90-9.20 ppm for $\alpha$-pyrone, peak at $\delta$ 6.30-7.20 ppm for aromatic protons.

Compound (IVa-j) have shown presence of a singlet between $\delta$ 9.10-10.9 ppm indicate the formation of oxime $(>\mathrm{C}=\mathrm{N}-\mathrm{OH}$ ) by simple condensation process in all the spectra has confirmed the formation of oxime derivative and remaining peak for rest of the structure have been observed similar to that of compound (IIIa-j).

In ${ }^{13} \mathrm{C}$ NMR the characteristic peaks of keto group at 160.90 ppm conforms the carbonyl group at pyranone ring, peaks appeared at 110-152 ppm showed the peaks of aromatic carbon in molecule, peaks at 21.20 conforms the methyl group on pyranone ring. Compound (IVa-j) have shown ${ }^{13} \mathrm{C}$ NMR data at 168.20 conforms the formation of oxime, remaining have appeared similar to the compounds for (IIIa-j). Final conformation of derivative have been done by DIPMS data, electron impact mass spectra showed an accurate molecular ion peak at $\mathrm{m} / \mathrm{z} 281.3256,297.3276,297.3276,297.3276$, $314.0127,314.0127,314.0127,326.0350,326.0350,325.0420$ for title compounds IIIa-j, respectively, and electron impact mass spectra showed an accurate molecular ion peak at $\mathrm{m} / \mathrm{z}$ $296.060,312.055,312.055,312.055,330.022,330.022$, $330.022,341.046,341.046$, and 340.052 for title compounds IVa-j, respectively.

The synthesized compounds were evaluated for in vitro antibacterial and antifungal activity against various strains $\operatorname{Gr}(+)$ bacterial strains: B. subtilis; S. aureus, $\operatorname{Gr}(-)$ bacterial strains: E. coli; P. aeruginosa, fungal strains: S. cerevisiae; $A$. niger, C. albicans using nutrient agar cup plate method. The results are given in Table 1 and 2 .

We studied the effect of various phenothiazine and its oxime derivatives of coumarins containing various substituents like nitro $\left(-\mathrm{NO}_{2}\right)$, chloro $(-\mathrm{Cl})$, hydroxyl $(-\mathrm{OH})$, carboxylic $(-$ 
$\mathrm{COOH}$ ) groups. The results showed that compounds (IIIa), (IIIe), (IIIh), (IIIj), (IVa), (IVi), and (IVj) exhibited comparable antibacterial and antifungal activity with the standard antibiotics ciprofloxin and greseofluvin. It has been observed that compound (IVa) show better antibacterial and antifungal activity compare to individual coumarin and phenothiazine and compounds (IIIa) and (IVa) (contains no substitution at ring 4) were shown better activity compare to rest of compounds with various substitution like $-\mathrm{OH},-\mathrm{Cl},-\mathrm{NO}_{2}$ and $-\mathrm{COOH}$ at different positions like ortho, meta, and para.

\section{Conclusions}

In this paper, we report the synthesis and antimicrobial activity of novel series of 4-methyl-2-oxo-pyranylphenothiazines (IIIa-j) and 4-methylpyrano-(2,3- $\beta$ )phenothiazine-2 $(11 H)$-one oxime (IVa-j). The preliminary in vitro antimicrobial activity of these novel series of derivative has evidenced that some of newly synthesized derivatives have shown very prominent potential as antimicrobial agents. The possible improvement of antimicrobial activity of these derivatives can be further modified based on modulation of ring substituent and/or additional fictionalization warrants further investigation. In summary, we have identified novel series of 4-methyl-2-oxo-pyranyl-phenothiazines (IIIa-j) and 4methylpyrano-(2,3- $\beta)$-phenothiazine-2 $(11 H)$-one oxime (IVa-j) which may develop into potential class of antimicrobial agents.

\section{Acknowledgement}

Authors are thankful to Dr. Vipin Bihari Gupta, director Bhanwar Nahata College of Pharmacy, Mandsaur (Madhya Pradesh) for providing necessary facilities.

\section{References}

[1]. Bisignano, G.; Sanogo, R.; Marino, A.; Aquino, R.; D'angelo, V.; Germanò, M.P.; De Pasquale, R.; Pizza, C. Lett. Appl. Microbiol. 2000, 30, 105-108.

[2]. Kayser, O.; Kolodziej, H. Planta Med. 1997, 63, 508-510.

[3]. Sardari, S.; Mori, Y.; Horita, K.; Micetich, R.G.; Nishibe, S.; Daneshtalab, M. Bioorg. Med. Chem. 1999, 7, 1933-1940.

[4]. Jurd, D. L.; Corse, J.; King, A.; Bayne, H.; Mihara, K. Phytochemistry 1971, 10, 2971-2974.

[5]. Jurd, D. L.; King, J. R.; Mihara, K. Experientia. 1970, 26, 1281-1283.

[6]. Shukla, Y.; Srivastava, A.; Kumar, S.; Kumar, S. J. Ethnopharm. 1999, 67, 241-245.

[7]. Kwon, Y. S.; Kobayashi, A.; Kajiyama, S. I.; Kawazu, K.; Kanzaki, H.; Kim, C. M. Phytochemistry 1997, 44, 887-889.

[8]. Marston, A.; Hostettmann, K.; Msonthi, J. D. J. Nat. Prod. 1995, 58, 128 130.

[9]. Fuller, R. W.; Bokesch, H. R.; Gustafson, K. R.; Mckee, T. C.; Cardellina, J. H.; Mcmahon, J. B.; Cragg, G. M.; Sojaerto, D. D.; Boyd, M. R. Bioorg. Med. Chem. Lettr. 1994, 4, 1961-1964

[10]. Yang, Y. Z.; Ranz, A.; Pan, H. Z.; Zhang, Z. N.; Lin, X. B.; Meshnick, S. R. Am. J. Trop. Med. Hyg. 1992, 46, 15-20.

[11]. Ehlich, P. Chemotherapeutics: scientific principles, methods, and results. Lancet. 1913, Ed. 2, pp. 445-451.

[12]. Leonard, A.; Jette, E. K. Int. J. Antimicrob. Agent. 2001, 18, 411-417.

[13]. Amaral, L.; Kristiansen, J.; Thomsen, V.; Markowich, B. Int. J. Antimicrob. Agents. 2000, 14, 225-229.

[14]. Ratnakar, P.; Rao, S. P.; Sriramarao, P. Int. Clin. Psychopharmocol. 1995, 10, 39-43.

[15]. Kristiansen, J. E.; Justesen, T.; Hvidberg, E. F.; Andersen, L. P. Pharmacol Toxicol. 1989, 64, 386-388.

[16]. Chakrabarty, A. N.; Bhattacharya, C. P.; Dasdidar, S. G. Acta Path et Microbiol Scand. 1993, 101, 449-454.

[17]. Amaral, L.; Kristiansen, J. E.; Lorian, V. J. Antimicrob. Chemother. 1992, 30, 556-558.

[18]. Bettencourt, V. M.; Amaral, L. Int. J. Antimicrob. Agents. 2001, 17, 225228.

[19]. Kristiansen, J. E.; Vergann, B. Acta Path. Micro. 1986, 94, 393-398

[20]. Molnar, J.; Beladi, I.; Foldes, I. Zbl. Bakt. Mik. Hyg. 1977, 239, 521-526.

[21]. Deeds, F.; Stockton, A. B.; Thomas, J. O. Pharmaco. Exp. Therapy 1939, 65, 353-371.

[22]. Chan, Y.; Ong, Y.; Chua, K. L. Antimicrob. Agents. Ch. 2007, 51, 623-630.
[23]. Desai, J. J.; Desai, P. G.; Mehta, A. G. Asian J. Chem. 1999, 11, 519-522.

[24]. Desai, J. J.; Desai, P. G.; Mehta, A. G. Asian J. Chem. 2000, 12, 1067.

[25]. Hania, M. M. Asian J. Chem. 2002, 14, 1074-1079.

[26]. Rai, B. K. Asian J. Chem., 2002, 14, 1595-1599.

[27]. Rai, B. K., Asian J. Chem. 2002, 14, 312-316.

[28]. Hania, M. M. Asian J. Chem. 2005, 17, 439-442.

[29]. Dobeck, A. S.; Klayman, D. Antimicrob Agents Chemother. 1980, 18, $27-$ 30.

[30]. Wagner, W. H.; Winkelman. E. Arzneim Forschi. 1972, 22, 1713-1721.

[31]. Hania, M. M. Asian J. Chem. 2007, 17, 439-443.

[32]. Morrison, N. E.; Collins, F. M. Int. J. Leprosy. 1981, 49, 180-188.

[33]. Jones, D. H.; Slack, R.; Squires, S. J. Med. Chem. 1965, 2, 676-681.

[34]. Klayman, D. L.; Joseph, F. J. Med. Chem. 1979, 22, 855-860.

[35]. Mann, F. G.; Saunders, B. C. Practical Organic Chemistry, Longan Inc. New York, 1960, $6^{\text {th }}$ Edn. pp 307-308.

[36]. Pandey, V. K.; Saxena, S. K.; Joshi, M. N. Indian J. Chem. 2004, 43, 10151017.

[37]. Li, J. J.; Limberakis, C.; Pflum, D. A. Modern Organic Synthesis in the Laboratory A Collection of Standard Experimental Procedures, Pfizer Global Research \& Development, Oxford University press, 2007, 2nd Edn., pp 42-44.

[38]. Hart, D. J.; Magomedov, N. A. J. Am. Chem. Soc. 2001, 123, 5892-5899.

[39]. Coleman, K. Drug Discov. Today, Therapeutic Strategies 2004, $1^{\text {st }}$ Edn. pp 455-460.

[40]. Smyth, R. D. Clinical analysis, Microbiology, Remington's Pharmaceutical sciences $18^{\text {th }}$ Edition, Mack Publishing Company Peninisilvenia, 1991, pp. 524-27

[41]. Biological assay, Indian Pharmacopoeia published by Govt. of India 1996 (2): A-88.

[42]. Pellczar, M. J. R.; Chan, E. C. S. Antibiotics and other chemotherapeutic agent Microbiology, TMH Edition, TATA-McGraw-Hill Publishing Houses, 1989, pp. 466-493

[43]. Harry, W. Antiseptic and Disinfectant action, Microbes in Action, A Laboratory manual in Microbiology 1982, pp. 75-76.

[44]. Davis, W. W.; Stout, T. R. Appl. Environ. Microbiol. 1971, 22(4), 666670.

[45]. Lalitha, M. K. Manual on Antimicrobial Susceptibility Testing (Under the auspices of Indian Association of Medical Microbiologists) http://www.ijmm.org/documents/Antimicrobial.doc.

[46]. Gaud, S. R. Microbial Assays, In Practical Microbiology, Nirali Publication, 2006, 2nd Edn. Pp. 111-116.

[47]. Carter, S. J. Basics of Microbiology, Cooper and Gunn's Tutorial Pharmacy, CBS Publishers and Distributors, 2005, pp. 289-366.

[48]. Andrews, J. M. J. Antimicrob. Chemoth. 2001, 48, 5-11.

[49]. Phair, J. P.; Watanakunakorn, C.; bannister, T. Am. Soc. Microb. 1969, 18(3), 303-306.

[50]. Therese, K. L.; Bagyalakshmi, R.; Madhavan, H. N.; Deepa, P. Indian J. Med. Microbio. 2006, 24(4), 273-279. 